\title{
The Herschel M 33 extended survey (HerM33es): PACS spectroscopy of the star forming region BCLMP $302^{\star}$ (Corrigendum)
}

\author{
B. Mookerjea ${ }^{1}$, C. Kramer ${ }^{2}$, C. Buchbender ${ }^{2}$, M. Boquien ${ }^{3}$, S. Verley ${ }^{4}$, M. Relaño ${ }^{4}$, G. Quintana-Lacaci ${ }^{2}$, \\ S. Aalto ${ }^{5}$, J. Braine ${ }^{6}$, D. Calzetti ${ }^{3}$, F. Combes ${ }^{7}$, S. Garcia-Burillo ${ }^{8}$, P. Gratier ${ }^{6}$, C. Henkel ${ }^{9}$, F. Israel ${ }^{10}$, S. Lord $^{11}$, \\ T. Nikola ${ }^{12}$, M. Röllig ${ }^{13}$, G. Stacey ${ }^{12}$, F. S. Tabatabaei ${ }^{9}$, F. van der Tak ${ }^{14}$, and P. van der Werf ${ }^{10,15}$ \\ 1 Tata Institute of Fundamental Research, Homi Bhabha Road, Mumbai 400005, India \\ e-mail: bhaswati@tifr.res.in \\ 2 Instituto Radioastronomía Milimétrica, Av. Divina Pastora 7, Nucleo Central, 18012 Granada, Spain \\ 3 University of Massachusetts, Department of Astronomy, LGRT-B 619E, Amherst, MA 01003, USA \\ 4 Universidad de Granada, 18012 Granada, Spain \\ 5 Department of Radio and Space Science, Onsala Observatory, Chalmers University of Technology, 43992 Onsala, Sweden \\ ${ }^{6}$ Laboratoire d'Astrophysique de Bordeaux, Université Bordeaux 1, Observatoire de Bordeaux, OASU, UMR 5804, CNRS/INSU, \\ BP 89, 33270 Floirac, France \\ 7 Observatoire de Paris, LERMA, CNRS, 61 Av. de l'Observatoire, 75014 Paris, France \\ 8 Observatorio Astronómico Nacional (OAN) - Observatorio de Madrid, Alfonso XII 3, 28014 Madrid, Spain \\ 9 Max Planck Institut für Radioastronomie, Auf dem Hügel 69, 53121 Bonn, Germany \\ ${ }^{10}$ Leiden Observatory, Leiden University, PO Box 9513, 2300 RA Leiden, The Netherlands \\ 11 IPAC, MS 100-22 California Institute of Technology, Pasadena, CA 91125, USA \\ 12 Department of Astronomy, Cornell University, Ithaca, NY 14853, USA \\ 13 KOSMA, I. Physikalisches Institut, Universität zu Köln, Zülpicher Strasse 77, 50937 Köln, Germany \\ 14 SRON Netherlands Institute for Space Research, Landleven 12, 9747 AD Groningen, The Netherlands \\ 15 SUPA, Institute for Astronomy, University of Edinburgh, Royal Observatory, Blackford Hill, Edinburgh EH9 3HJ, UK
}

A\&A 532, A152 (2011), DOI: 10.1051/0004-6361/201116447

Key words. local group - HII regions - galaxies: individual: M 33 - photon-dominated region (PDR) - galaxies: ISM errata, addenda

\section{1. $[\mathrm{CII}] / \mathrm{CO}(1-0)$ intensity ratio}

We made an error in the calculation of $\left[\mathrm{C}_{\mathrm{II}}\right] / \mathrm{CO}(1-0)$ intensity ratios presented in our recent publication (Mookerjea et al. 2011). We assumed a $\mathrm{CO}(2-1) / \mathrm{CO}(1-0)$ intensity ratio of 0.8 on the $\mathrm{K} \mathrm{km} \mathrm{s}^{-1}$ scale (Gratier et al. 2010), but calculated the $\mathrm{CO}(1-0)$ intensities on the erg s $\mathrm{sm}^{-1} \mathrm{sr}^{-1}$ scale incorrectly. The resulting $\left[\mathrm{C}_{\mathrm{II}}\right] / \mathrm{CO}(1-0)$ ratios are too low by a factor 8.0. This affects Fig. 12 and one sentence of the last paragraph of the Discussion. The values used for the $\mathrm{H} \alpha$ peak position presented in Sect. 6, are correct as they were derived directly from observed $\mathrm{CO}(1-0)$ spectra. The corrected figure and text are given below.

In BCLMP 302/M33, we find that the $\left[\mathrm{C}_{\mathrm{II}}\right] / \mathrm{CO}(1-0)$ ratio varies between $10^{3}$ and $7 \times 10^{4}$ (Fig. 12), with the $\mathrm{H}$ II region $\mathrm{A}$ shown by red markers having values in the range $6 \times 10^{3}$ to $3 \times 10^{4}$. The spiral arm region $\mathrm{B}$ (green markers) exhibits values in the range $1 \times 10^{3}$ to $4 \times 10^{3}$, while region $\mathrm{C}$ (cyan markers), where $\mathrm{CO}$ has hardly been detected, shows higher values, between $6 \times 10^{3}$ and $7 \times 10^{4}$.

\footnotetext{
* Herschel is an ESA space observatory with science instruments provided by European-led Principal Investigator consortia and with important participation from NASA.
}

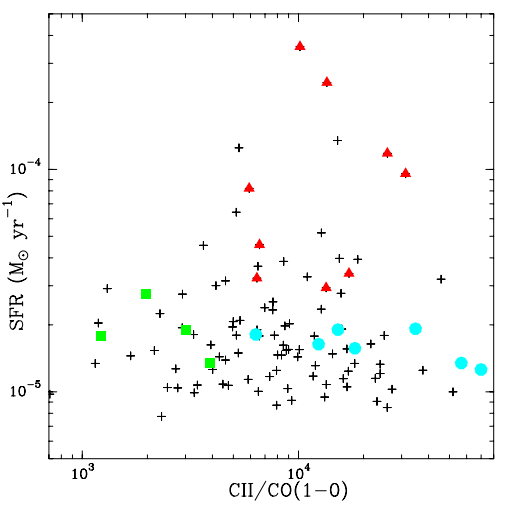

Fig. 12. Star formation rate (SFR) versus the $\left[\mathrm{C}_{\mathrm{II}}\right] / \mathrm{CO}(1-0)$ intensity ratio. Markers are the same as in Fig. 7.

Figures 7 and 12 show values on a $12^{\prime \prime}$ grid, and not on a $6^{\prime \prime}$ grid as mentioned in the caption of Fig. 7 of the published paper.

\section{References}

Gratier, P., Braine, J., Rodriguez-Fernandez, N. J., et al. 2010, A\&A, 522, A3 Mookerjea, B., Kramer, C., Buchbender, C., et al. 2011, A\&A, 532, A152 